\title{
s \\ Modulation doping of a Mott quantum well by a proximate polar discontinuity
}

\author{
T. Higuchi, ${ }^{1,}$ ' Y. Hotta, ${ }^{1}$ T. Susaki, ${ }^{1}$ A. Fujimori, ${ }^{2}$ and H. Y. Hwang ${ }^{1,3}$ \\ ${ }^{1}$ Department of Advanced Materials Science, University of Tokyo, Kashiwa, Chiba 277-8561, Japan \\ ${ }^{2}$ Department of Physics, University of Tokyo, Bunkyo-ku, Tokyo 113-0033, Japan \\ ${ }^{3}$ Japan Science and Technology Agency, Kawaguchi 332-0012, Japan
}

(Received 28 April 2008; published 9 February 2009)

\begin{abstract}
We present evidence for hole injection into $\mathrm{LaAlO}_{3} / \mathrm{LaVO}_{3} / \mathrm{LaAlO}_{3}$ quantum wells near a polar surface of $\mathrm{LaAlO}_{3}(001)$. As the surface is brought in proximity to the $\mathrm{LaVO}_{3}$ layer, an exponential drop in resistance and a decreasing positive Seebeck coefficient are observed below a characteristic coupling length of 10-15 unit cells. We attribute this behavior to a crossover from an atomic reconstruction of the $\mathrm{AlO}_{2}$-terminated $\mathrm{LaAlO}_{3}$ surface to an electronic reconstruction of the vanadium valence. These results suggest a general approach to tunable hole doping in oxide thin-film heterostructures.
\end{abstract}

DOI: 10.1103/PhysRevB.79.075415

PACS number(s): 73.40.-c, 71.28. $+\mathrm{d}, 71.30 .+\mathrm{h}, 73.50 . \mathrm{Lw}$

\section{INTRODUCTION}

Surfaces and interfaces of oxides have been of growing interest partially because of the rich variety of bulk oxide functionalities, ${ }^{1}$ as well as their unique reconstruction mechanisms ${ }^{2-4}$ not found in conventional semiconductors. ${ }^{5,6}$ The observation of metallic interfaces between two perovskite insulators, $\mathrm{LaAlO}_{3}$ and $\mathrm{SrTiO}_{3},{ }^{2}$ has motivated many studies on the origin of this conductivity. Two scenarios have been proposed, one is based on electronic reconstructions driven by the polar discontinuity at the interface ${ }^{7-9}$ and another is based on growth-induced oxygen vacancies. ${ }^{10,11}$

The polar discontinuity scenario examines the built-in charge structure at the interface. From an ionic point of view, $\mathrm{LaAlO}_{3}$ is polar along the (001) direction with alternate stacking of $(\mathrm{LaO})^{+}$and $\left(\mathrm{AlO}_{2}\right)^{-}$layers, while $\mathrm{SrTiO}_{3}$ is nonpolar with $(\mathrm{SrO})^{0}$ and $\left(\mathrm{TiO}_{2}\right)^{0}$ layers. When one unit cell (uc) of $\mathrm{LaAlO}_{3}$ is placed on $\mathrm{SrTiO}_{3}$, there is a dipole shift in the electrostatic potential. Additional $\mathrm{LaAlO}_{3}$ layers build up this dipole shift, leading to a diverging potential in the limit of infinite $\mathrm{LaAlO}_{3}$ thickness. To prevent this catastrophic situation, injection of $-q / 2$ (where $q$ is the elementary charge) per two-dimensional (2D) uc is needed at the $\left(\mathrm{AlO}_{2}\right)^{-}-(\mathrm{LaO})^{+}-\left(\mathrm{TiO}_{2}\right)^{0}-(\mathrm{SrO})^{0}$ interface, which can be accommodated by a partial valence change of $\mathrm{Ti}^{4+}$ to $\mathrm{Ti}^{3+}$ near the interface. The $\mathrm{Ti}^{3+}$ component provides mobile $\mathrm{SrTiO}_{3}$ conduction electrons in this picture. ${ }^{7}$ Alternatively, it has been proposed that the essential origin of the conducting interface is the formation of $\mathrm{SrTiO}_{3}$ surface oxygen vacancies during the highly kinetic growth of films by pulsed laser deposition (PLD). ${ }^{10,11} \mathrm{SrTiO}_{3}$ is known as a material which easily accommodates oxygen vacancies that readily dope itinerant electrons. ${ }^{12}$ Discriminating between these two proposed scenarios has been controversial in part because both mechanisms could give similar transport and spectroscopic signatures.

The $\mathrm{LaAlO}_{3} / \mathrm{SrTiO}_{3}$ system actually involves two polar discontinuities - the interface just described, as well as the polar $\mathrm{AlO}_{2}$-terminated surface of $\mathrm{LaAlO}_{3}$, which requires a net of $+q / 2$ per $2 \mathrm{D}$ uc. Recently it was found that the conducting $\mathrm{LaAlO}_{3} / \mathrm{SrTiO}_{3}$ interface exhibited a transition to an insulating state when the $\mathrm{LaAlO}_{3}$ was thinner than a critical thickness of four uc, bringing the two polar discontinuities close together. ${ }^{9}$ (A similar tendency was also observed for proximity coupling of two $\mathrm{LaAlO}_{3} / \mathrm{SrTiO}_{3}$ interface polar discontinuities with opposite sign. $^{8}$ ) This critical thickness may be interpreted as the threshold dipole shift below which it is energetically favorable to remain in an unreconstructed state for both the surface and the interface. ${ }^{13} \mathrm{~A}$ different but equivalent perspective is that the polar $\mathrm{AlO}_{2}$-terminated surface of $\mathrm{LaAlO}_{3}$ is compensating the electrons at the $\mathrm{LaAlO}_{3} / \mathrm{SrTiO}_{3}$ interface by hole doping on short length scales. Far above the critical thickness, the polar $\mathrm{LaAlO}_{3}$ (001) surface atomically reconstructs via surface off stoichiometry and surface relaxation; electronic reconstructions are unavailable because of the fixed valence of $\mathrm{La}, \mathrm{Al}$, and O. ${ }^{14-16}$ Below a characteristic coupling distance, however, hole doping provides an alternative electronic reconstruction if it is energetically favorable.

In order to test this possibility of hole doping, we have studied the transport properties of the Mott insulator $\mathrm{LaVO}_{3}$ embedded in $\mathrm{LaAlO}_{3}$ in trilayer structures [Fig. 1(a)]. $\mathrm{LaVO}_{3}$ is an attractive candidate since it can be readily hole doped by chemical substitution ${ }^{17}$ and because of its structural and thermodynamic compatibility with $\mathrm{LaAlO}_{3}$ for the growth of atomically precise thin-film structures. Although $\mathrm{LaVO}_{3}$ has the same polar structure as $\mathrm{LaAlO}_{3}$, and hence no polar discontinuity at their interface, we find an exponential drop in resistance when a polar $\mathrm{AlO}_{2}$-terminated surface of $\mathrm{LaAlO}_{3}$ is brought in close proximity to the $\mathrm{LaVO}_{3}$ quantum well. Furthermore, the positive thermopower voltage measured indicates holelike carriers, which scales with the doping dependence of bulk $\mathrm{LaVO}_{3}$. These results indicate that polar discontinuities can be utilized for the tunable doping of holes, which cannot arise by growth-induced oxygen vacancies.

\section{METHODS}

$\mathrm{LaAlO}_{3}(n \mathrm{uc}) / \mathrm{LaVO}_{3}(m \mathrm{uc}) / \mathrm{LaAlO}_{3}$ (substrate) heterostructures $[\mathrm{LAO}(n) / \mathrm{LVO}(m)]$ were fabricated by PLD using a $\mathrm{KrF}$ excimer laser with a laser fluence of $1.0 \mathrm{~J} / \mathrm{cm}^{2}$, a spot size of $1.6 \mathrm{~mm}^{2}$, and repetition rate of $8 \mathrm{~Hz}$. The thickness of each layer was monitored by RHEED oscillations [Fig. 
(a)
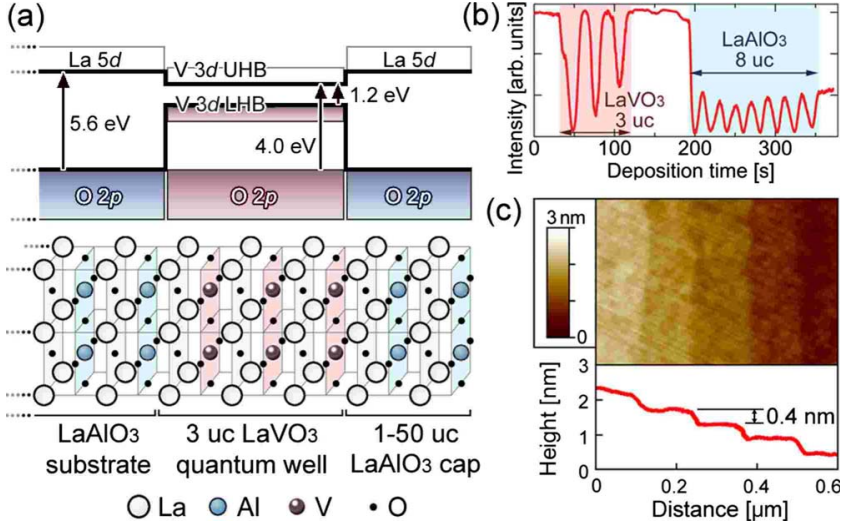

FIG. 1. (Color online) (a) Schematic band and crystal structure of $\mathrm{LAO}(n) / \mathrm{LVO}(3)$ quantum wells grown on $\mathrm{AlO}_{2}$-terminated $\mathrm{LaAlO}_{3}(001)$ substrates. Filled and empty bands correspond to the valence and conduction bands, respectively, and $\mathrm{O} 2 p$ bands are assumed to be aligned. (b) Typical reflection high-energy electrondiffraction (RHEED) oscillations during growth of LAO(8)/ LVO(3). (c) Atomic force microscopy (AFM) image of LAO(50)/ $\mathrm{LVO}(3)$ showing a clear step and terrace surface with step height of $\sim 0.4 \mathrm{~nm}$.

1(b)]. $\quad \mathrm{AlO}_{2}$-terminated $\quad \mathrm{LaAlO}_{3} \quad$ (001) single-crystal substrates ${ }^{18}$ were preannealed at $950{ }^{\circ} \mathrm{C}$ for $30 \mathrm{~min}$, and $m$ uc of $(m=1-30) \mathrm{LaVO}_{3}$ layers were deposited followed by $n$ uc of $(n=1-50) \mathrm{LaAlO}_{3}$ capping layers using $\mathrm{LaVO}_{4}$ polycrystalline and $\mathrm{LaAlO}_{3}$ single-crystal targets, respectively. The growth temperature was $600{ }^{\circ} \mathrm{C}$ and the oxygen partial pressure was $1.0 \times 10^{-6}$ Torr for all processes. These conditions follow our previous optimization of high-quality $\mathrm{LaVO}_{3}$ thin-film growth in the layer-by-layer growth mode ${ }^{19}$ with one exception. Note the different laser conditions: we now use a four lens afocal zoom stage to accurately image an aperture rather than a single lens just off of the focusing condition. This is far less sensitive to the divergence characteristics of the laser, and hence much more reproducible between systems. We confirmed that one RHEED oscillation corresponds to the formation of one perovskite unit cell by characterizing the superlattices of $\mathrm{LaAlO}_{3}$ and $\mathrm{LaVO}_{3}$ using $\mathrm{x}$-ray diffraction.

The stoichiometry of $\mathrm{LaVO}_{3}$ films grown in these conditions has been confirmed from several different perspectives in previous studies. The film lattice volume was found to be close to bulk (accounting for the compressive strain by the substrate) - usually, in the presence of significant cation defects, there is a significant expansion of the lattice. ${ }^{19} \mathrm{LaVO}_{3}$ films were also studied extensively by scanning transmission electron microscopy and electron energy-loss spectroscopy (EELS). ${ }^{20}$ The insulating $\mathrm{LaVO}_{3}$ films have a clear $\mathrm{V}^{3+}$ valence, as observed in the $\mathrm{V}-L_{2,3}$ edge. Any significant doping by off stoichiometry would have shown clear deviations from $\mathrm{V}^{3+}$ spectra. Thus the stoichiometry of our $\mathrm{LaVO}_{3}$ films has been well established in these previous studies. $\mathrm{LaAlO}_{3} / \mathrm{LaVO}_{3} / \mathrm{LaAlO}_{3}$ quantum wells could not be grown at higher oxygen partial pressures than those used here due to the competing formation of polycrystalline $\mathrm{LaV}^{5+} \mathrm{O}_{4} \cdot{ }^{19,20}$ Furthermore, the $\mathrm{LaVO}_{3}$ layers in the as-grown quantum wells converted to the insulating transparent $\left(d^{0}\right) \mathrm{LaV}^{5+} \mathrm{O}_{4}$ phase upon oxygen post annealing.

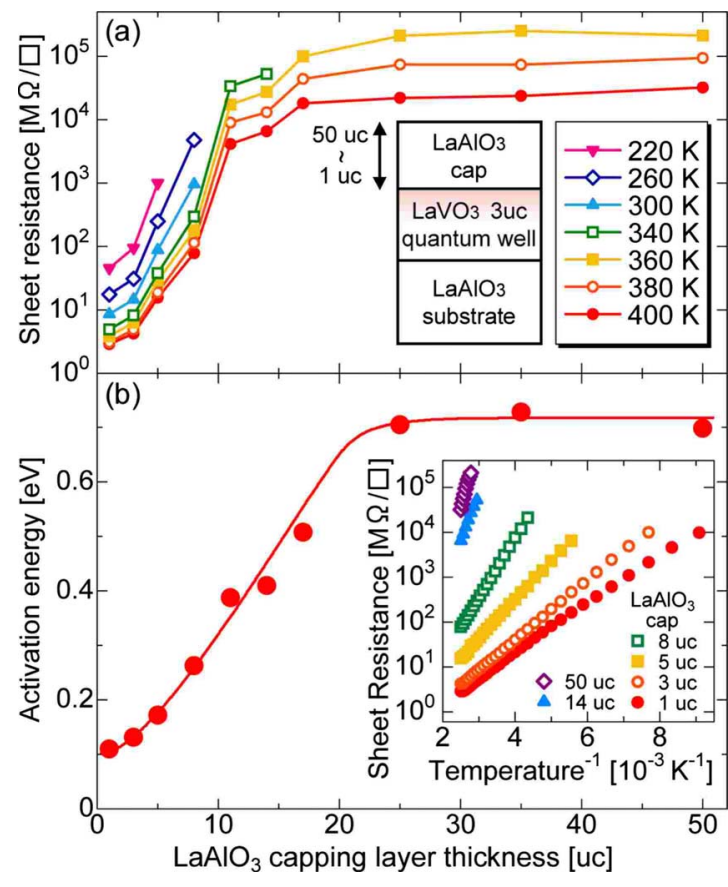

FIG. 2. (Color online) (a) Sheet resistance and (b) activation energy of $\mathrm{LAO}(n) / \mathrm{LVO}(3)$ as a function of $\mathrm{LaAlO}_{3}$ cap thickness $n$. The curve is a guide for the eyes. Inset of (b) shows Arrhenius plots of the sheet resistance.

The film surface was investigated by AFM, and all structures were atomically flat with a clear step and terrace structure reflecting the slight miscut angle of the substrate [Fig. 1(c) $]$. The step size $(\sim 0.4 \mathrm{~nm})$ was consistent with the height of one $\mathrm{LaAlO}_{3}$ uc (pseudocubic lattice constant $\left.a_{\mathrm{LaAlO}_{3}}=0.379 \mathrm{~nm}\right)$. Ohmic contacts were made to the buried quantum well layers using indium ultrasonic soldering, which penetrated more than $40 \mathrm{~nm}$ from the surface as confirmed using buried $\mathrm{SrVO}_{3}$ test structures.

\section{RESULTS AND DISCUSSION}

\section{A. $\mathrm{LaAlO}_{3}$ cap thickness dependence}

The in-plane sheet resistance was dramatically dependent on the $\mathrm{LaAlO}_{3}$ cap thickness as shown in Fig. 2(a), where the thickness of the $\mathrm{LaVO}_{3}$ layer was fixed to three uc. Below a characteristic thickness of around 10-15 uc, the sheet resistance decreased exponentially. For all samples the sheet resistance showed Arrhenius-type thermally activated behavior as shown in the inset of Fig. 2(b). The activation energy was $\sim 0.7 \mathrm{eV}$ for the thick capping layer samples and decreased to $\sim 0.1 \mathrm{eV}$ with decreasing thickness. Since $\mathrm{LaAlO}_{3}$ is such a robust insulator (both in bulk and as measured in our thin films), the resistance of the embedded $\mathrm{LaVO}_{3}$ layer could be measured to the very high values shown.

Similar results were found for these $\operatorname{LAO}(n) / \operatorname{LVO}(3)$ trilayer structures further capped with a nonpolar material, $10 \mathrm{uc}$ of $\mathrm{SrTiO}_{3}$. Here the measurements were limited to a lower range of resistance values $(n \leq 8)$ by the higher intrinsic conductivity of $\mathrm{SrTiO}_{3}$. This suggests that the conductivity of the $\mathrm{LaVO}_{3}$ layer only depends on the distance 


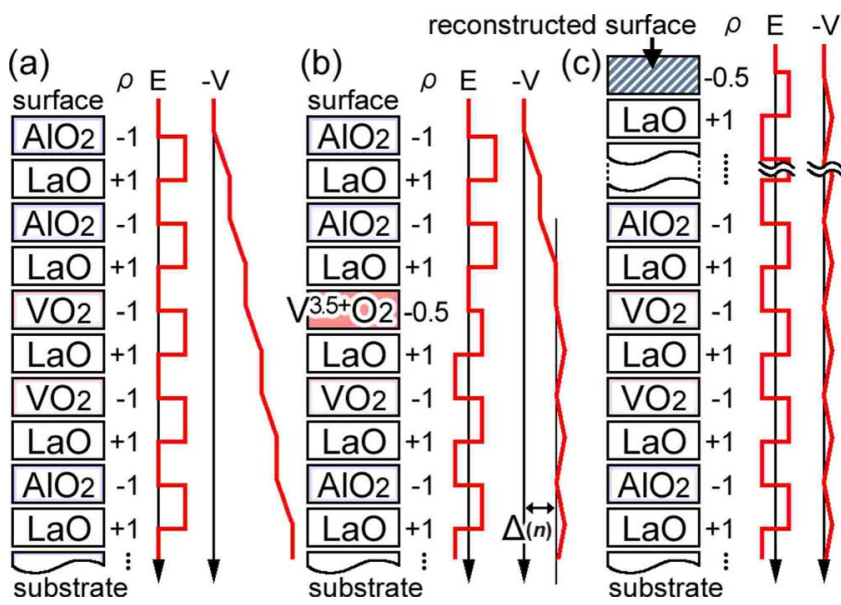

FIG. 3. (Color online) Schematic diagrams of possible reconstruction processes. (a) Without reconstruction the structure is composed of negatively and positively charged $(\rho)$ layers from the surface, which induces a non-negative electric field $(E)$, leading to a divergence in potential $(-V)$. (b) Electronic reconstruction at the $\mathrm{LaVO}_{3}$ quantum well layer with a net half hole per 2D unit cell induced. The potential divergence is canceled, but a finite shift $\Delta(n)$ in potential remains. (c) Atomic reconstruction of the $\mathrm{LaAlO}_{3}$ polar surface.

to the polar surface $\left(\mathrm{LaAlO}_{3}\right.$ surface) or interface $\left(\mathrm{SrTiO}_{3} / \mathrm{LaAlO}_{3}\right)$ and not on the total thickness of the material deposited on top of it. Therefore, the role of $\mathrm{LaVO}_{3}$ defects created during growth of the cap can be neglected here.

These results are well explained by the electrostatic coupling of reconstructions of the polar surface and the quantum well, as illustrated in Fig. 3. Since we fabricated the quantum well structures by perovskite unit-cell deposition on $\mathrm{AlO}_{2}$-terminated $\mathrm{LaAlO}_{3}$ substrates, the surface of the $\mathrm{LaAlO}_{3}$ cap preserves $\mathrm{AlO}_{2}$ termination, which requires a net of $+q / 2$ per $2 \mathrm{D}$ uc to avoid the potential divergence arising from the surface polar discontinuity. Here we consider two reconstruction mechanisms for this system. The first possibility is hole injection into the $\mathrm{LaVO}_{3}$ quantum well layer. In this case we have no divergence but still a finite dipole shift $\Delta(n)$ arising from the polar $\mathrm{LaAlO}_{3}$ cap. Thus the total energy of this electronic reconstruction is the sum of the energy cost to change the vanadium valence $E_{\mathrm{val}}$ and $\Delta(n)$. The second process is the normal surface reconstruction of $\mathrm{LaAlO}_{3}$ where an atomic reconstruction is dominant (oxygen vacancies and lattice distortions) to provide positive charge. This reconstruction requires an energy cost $E_{\text {sur }}$ which is nominally independent of the thickness of the cap layer. Note that this is the same $E_{\text {sur }}$ calculated in Ref. 21 with respect to $E_{\mathrm{val}}$ for $\mathrm{Ti}$, where the conducting $\mathrm{LaAlO}_{3} / \mathrm{SrTiO}_{3}$ interface is toggled by manipulating the surface reconstruction. The difference in $E_{\mathrm{val}}$ for $\mathrm{Ti}$ and $\mathrm{V}$ leads to the different critical thickness observed in the two systems.

In the simplest form, $\Delta(n)$ increases linearly as a function of the $\mathrm{LaAlO}_{3}$ cap thickness $\Delta(n)=n q / 2 \varepsilon a_{\mathrm{LaAlO}_{3}}$ ( $\varepsilon$ is the dielectric constant of $\mathrm{LaAlO}_{3}$ ). If the cap is sufficiently thin (and $E_{\mathrm{val}}<E_{\text {sur }}$ ), then $E_{\mathrm{val}}+\Delta(n)<E_{\text {sur }}$ and hole injection is dominant, which decreases the resistance of the quantum

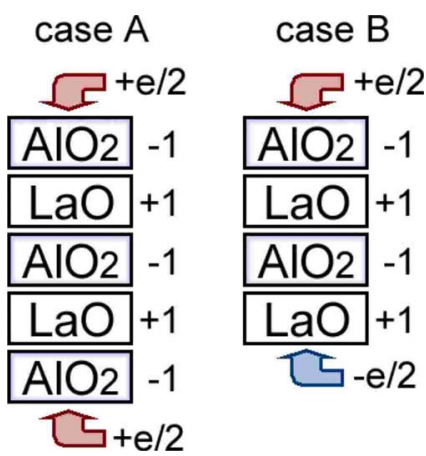

FIG. 4. (Color online) Schematic reconstructions of the top and bottom polar surfaces of (001)-oriented $\mathrm{LaAlO}_{3}$ for two different terminations of the bottom surface.

well. On the other hand, when the cap is very thick, $E_{\mathrm{val}}$ $+\Delta(n)>E_{\text {sur }}$, and the surface of the $\mathrm{LaAlO}_{3}$ cap is reconstructed, while the $\mathrm{LaVO}_{3}$ layers are undoped and insulating. Between these limiting cases, hole injection into the $\mathrm{LaVO}_{3}$ layer gradually decreases and the resistance increases as a function of the cap thickness, as observed in the transport measurements. Taking the $\mathrm{LaAlO}_{3}$ bulk dielectric constant $\varepsilon=24 \varepsilon_{0}\left(\varepsilon_{0}\right.$ is the vacuum permittivity), the dipole shift is $\Delta(10)=9.9 \mathrm{eV}$, exceeding the $\mathrm{LaAlO}_{3}$ bulk band gap of $\sim 5.6 \mathrm{eV}$. In the actual system this is greatly reduced by the strong polarization of the $\mathrm{LaAlO}_{3}$ lattice as observed by surface x-ray diffraction in $\mathrm{LaAlO}_{3}$ thin films on $\mathrm{SrTiO}_{3} .{ }^{22}$ Thus the detailed response is far more complex than that represented in Fig. 3, and the energy cost $\Delta(n)$ is likely dominated by the polarization energy of $\mathrm{LaAlO}_{3}$. A further discussion of the contrast in $\mathrm{LaAlO}_{3}$ thickness dependence of the conductivity observed here (conductivity in the thin limit), with prior results for the (001)-oriented $\mathrm{LaAlO}_{3} / \mathrm{SrTiO}_{3}$ and $\mathrm{LaVO}_{3} / \mathrm{SrTiO}_{3}$ interfaces ${ }^{9,23}$ (conductivity in the thick limit), is given in the Appendix.

Note that Fig. 3 only shows the near surface region with the $\mathrm{LaVO}_{3}$ quantum well. To formally confirm that global charge neutrality is maintained for the total system in all cases of reconstructions, the structure of the bottom surface must be known. This can best be illustrated in the schematic shown in Fig. 4, where we discuss only polar surfaces of $\mathrm{LaAlO}_{3}$ for simplicity.

As is well established in surface science, the polar surfaces are unstable to reconstructions driven to keep the electrostatic potential bounded. For case A, each surface requires $+e / 2$ charge to achieve this. The net $+e$ is compensated by the extra $\left(\mathrm{AlO}_{2}\right)^{-}$layer in the total structure, thus preserving global charge neutrality. For case B, the top surface requires $+e / 2$ charge, the bottom surface $-e / 2$ charge, and since the number of $\left(\mathrm{AlO}_{2}\right)^{-}$layers and $(\mathrm{LaO})^{+}$layers are equal, here again global charge neutrality is conserved. Note that in case $\mathrm{B}$, one could say that the $e / 2$ was transferred from the bottom to the top surface, while in case A, e/2 may be considered to arise locally. In either case, the reconstruction of the top $\left(\mathrm{AlO}_{2}\right)^{-}$surface is identical, although to formally conserve total charge, knowledge of the bottom interface is needed. Practically, these two surfaces are completely decoupled (the thickness of our substrates is $0.5 \mathrm{~mm}$, and certainly changing the termination layer on one surface does not affect 

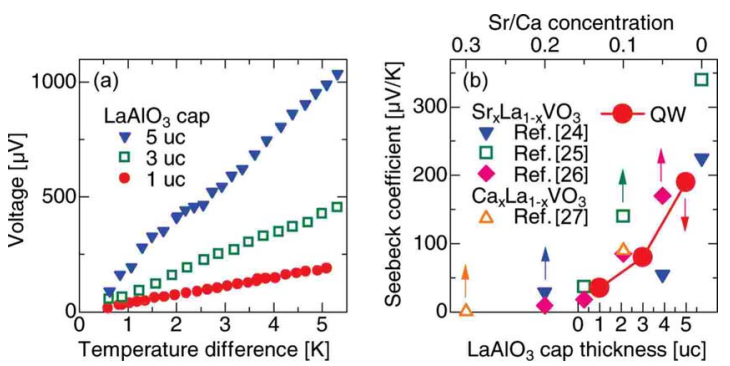

FIG. 5. (Color online) (a) Thermoelectric voltage measured as a function of applied temperature difference between two electrodes on $\mathrm{LAO}(n) / \mathrm{LVO}(3)$ quantum wells at room temperature. (b) Seebeck coefficient of $\operatorname{LAO}(n) / \operatorname{LVO}(3)$ as a function of $n$ (filled circles), plotted in comparison with bulk values for $\mathrm{La}_{1-x} \mathrm{Sr}_{x} \mathrm{VO}_{3}$ (Refs. 24-26) and $\mathrm{La}_{1-x} \mathrm{Ca}_{x} \mathrm{VO}_{3}$ (Ref. 27) at $300 \mathrm{~K}$.

the other across this macroscopic distance), so we address only the top surface in Fig. 3, as is conventional in such discussions of polar surfaces. Therefore our conclusions are independent of the assignment of charge transfer. Only when polar surfaces are microscopically close $e^{8,9,23}$ do they couple.

\section{B. Thermoelectric power}

A critical test of the model of coupled surface and interface reconstructions is whether the carriers induced by the electronic reconstruction are holes and not electrons as in all previous examples. ${ }^{2,7-11}$ They should further be tunable with the $\mathrm{LaAlO}_{3}$ cap layer thickness. To determine the sign of the transport carriers, the Seebeck coefficient $S$ of the $\mathrm{LAO}(n) / \mathrm{LVO}(3)$ structures was measured, as shown in Fig. 5. Intrinsic voltage fluctuations at high impedance limited the measurements to higher temperatures and thin $\mathrm{LaAlO}_{3}$ cap samples. The positive sign of $S$ confirms hole doping, and it systematically increased as a function of increasing $\mathrm{LaAlO}_{3}$ capping layer thickness, indicating a decreasing hole density and mirroring the evolution of the sheet resistance shown in Fig. 2. These results agree well with thermopower measurements of bulk $\mathrm{La}_{1-x} \mathrm{Sr}_{x} \mathrm{VO}_{3}$ and $\mathrm{La}_{1-x} \mathrm{Ca}_{x} \mathrm{VO}_{3}$, where $S$ was positive for $0<x<0.2$ and decreased as the dopant concentration $x$ increased. ${ }^{24-27}$ Given the compressive strain arising from the $\mathrm{LaAlO}_{3}$ substrate, the reduced electronic bandwidth, and interface scattering, a comparison between the $\mathrm{LaVO}_{3}$ quantum wells and bulk values is an approximate one. Nevertheless, this comparison indicates that the maximum equivalent hole density achieved in the single uc $\mathrm{LaAlO}_{3}$ cap sample is just below the bulk metal-insulator transition occurring at $x=0.18$ in $\mathrm{La}_{1-x} \mathrm{Sr}_{x} \mathrm{VO}_{3} \cdot{ }^{17} \mathrm{~A}$ recent theoretical proposal suggests that doped holes at $\mathrm{LaVO}_{3}$ interfaces are susceptible to charge ordering due to the artificial confined geometry, ${ }^{28}$ which may be relevant here.

\section{C. $\mathrm{LaVO}_{3}$ thickness dependence}

In the regime of a thin $\mathrm{LaAlO}_{3}$ cap layer, a V $\mathrm{V}^{4+}$ component giving rise to these holes should be observable. This has indeed been seen recently by x-ray photoemission spectroscopy on $\mathrm{LAO}(3) / \mathrm{LVO}(m)$ structures (grown on $\mathrm{SrTiO}_{3}$ substrates). ${ }^{29,30}$ In particular, the spatial distribution was

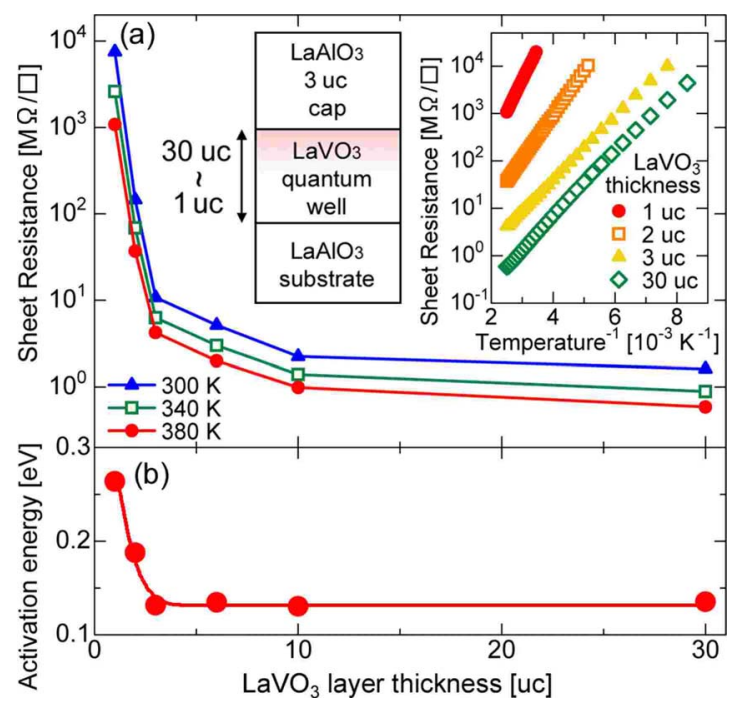

FIG. 6. (Color online) (a) Sheet resistance and (b) activation energy of $\mathrm{LAO}(3) / \mathrm{LVO}(m)$ as functions of $\mathrm{LaVO}_{3}$ layer thickness $m$. Inset of (a) shows Arrhenius plots of the sheet resistance. The curve is a guide for the eyes.

highly asymmetric, with the $\mathrm{V}^{4+}$ predominantly in the topmost $\mathrm{LaVO}_{3}$ layer. This nonuniformity naturally arises in Fig. 3(b) since $\Delta(n)$ would thus be minimized. The detailed charge distribution is a balance between $\Delta(n)$ and the electronic compressibility. This issue was further studied by measuring the transport properties of $\mathrm{LAO}(3) / \mathrm{LVO}(m)$ quantum well structures. As shown in Fig. 6, the sheet resistance was strongly dependent on the $\mathrm{LaVO}_{3}$ layer thickness $m$. The data did not scale with $1 / m$, as would be expected for a uniform three-dimensional (3D) resistivity. For $m>10$, there was little change in the sheet resistance, but for $m<6$ it increased more rapidly than $1 / m$, indicating the length scale for strong perturbation of the charge distribution by confinement effects. Thus the thickness-dependent resistance indicates that the conducting holes were distributed primarily at the top of the $\mathrm{LaVO}_{3}$ layer, as would be expected for doping arising from the polar $\mathrm{LaAlO}_{3}$ surface.

\section{SUMMARY}

We have found a strong electrostatic coupling between the $\mathrm{AlO}_{2}$-terminated $\mathrm{LaAlO}_{3}$ (001) surface and an embedded $\mathrm{LaVO}_{3}$ quantum well. When they are separated by less than 4-6 nm, transport measurements indicate a systematically increasing hole doping with decreasing separation. We propose that these results reflect a competition between atomic and electronic reconstructions, driven by the need to resolve the divergent surface energy arising from the polar surface termination. An important aspect of this study is that the electronic reconstruction involves holes and not electrons. Therefore the role of polar discontinuities can be more clearly distinguished from that of electron-donor oxygen vacancies, in contrast to previous examples. They further demonstrate that oxide heterostructures can be designed to introduce carriers without local chemical substitution, in analogy 
to modulation doping in compound semiconductor heterostructures.

\section{ACKNOWLEDGMENTS}

We thank Y. Hikita, K. Itaka, and T. Higuchi (Sr.) for helpful discussions. We acknowledge support from a Grantin-Aid for Scientific Research on Priority Areas. Y.H. acknowledges partial support from QPEC, Graduate School of Engineering, University of Tokyo.

\section{APPENDIX: COMPARISON OF $\mathrm{LaAlO}_{3}$ THICKNESS- DEPENDENT CONDUCTIVITY IN $\mathrm{LaAlO}_{3} / \mathrm{LaVO}_{3} / \mathrm{LaAlO}_{3}$ (001) WITH $\mathrm{LaAlO}_{3} / \mathrm{SrTiO}_{3}(001) \mathrm{AND}_{\mathrm{LaVO}} / \mathrm{SrTiO}_{3}$ (001)}

It is worth contrasting the $\mathrm{LaAlO}_{3}$ thickness dependence for the interface hole doping we find here, with prior results for electron doping at the (001)-oriented $\mathrm{LaAlO}_{3} / \mathrm{SrTiO}_{3}$ interface ${ }^{9}$ [and (001)-oriented $\mathrm{LaVO}_{3} / \mathrm{SrTiO}_{3}$ interface ${ }^{23}$ ]. For the $\mathrm{LaAlO}_{3} / \mathrm{SrTiO}_{3}$ structures, there are two polar discontinuities: the $\mathrm{LaAlO}_{3}$ surface and the $\mathrm{LaAlO}_{3} / \mathrm{SrTiO}_{3}$ interface. Without any reconstruction, the finite shift $\Delta$ remains, as shown in Fig. 7(a). When the $\mathrm{LaAlO}_{3}$ layer is thick, the large $\Delta$ makes the system unstable, and the polar discontinuities are reconstructed in order to solve the instability [the $\mathrm{LaAlO}_{3}$ surface as shown in Fig. 7(b)], and the $\mathrm{LaAlO}_{3} / \mathrm{SrTiO}_{3}$ interface by inducing $\mathrm{Ti}$ valence changes and associated metallic behavior as shown in Fig. 7(c). However, one reconstruction just by the $\mathrm{LaAlO}_{3}$ surface or by the $\mathrm{LaAlO}_{3} / \mathrm{SrTiO}_{3}$ interface alone is insufficient and the electrostatic potential diverges. Therefore, when the two polar discontinuities are far apart from each other and the potential shift $\Delta$ in Fig. 7(a) becomes large, both must reconstruct simultaneously, as shown in Fig. 7(d). Only when they are brought close together can they couple and remain (a)
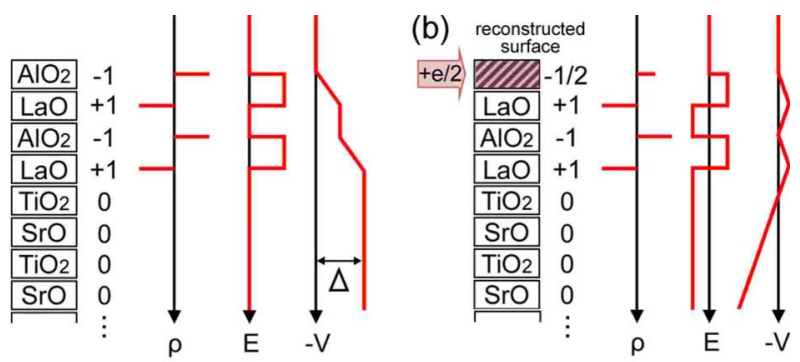

(c)

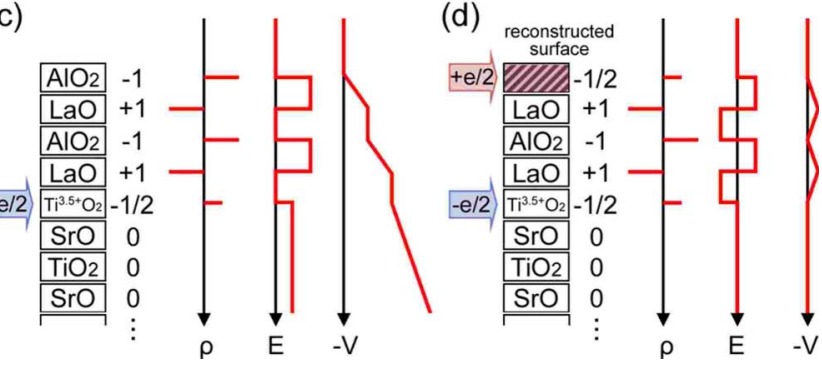

FIG. 7. (Color online) Schematic reconstructions of the polar surface of (001)-oriented $\mathrm{LaAlO}_{3}$ and the $\mathrm{LaAlO}_{3} / \mathrm{SrTiO}_{3}$ interface. (a) No reconstruction, (b) only the $\mathrm{LaAlO}_{3}$ surface is reconstructed (c) only the $\mathrm{LaAlO}_{3} / \mathrm{SrTiO}_{3}$ interface is reconstructed, and (d) both are reconstructed.

unreconstructed. ${ }^{8,9,23}$ Thus the $\mathrm{LaAlO}_{3} / \mathrm{SrTiO}_{3}$ interface is insulating for thin $\mathrm{LaAlO}_{3}$ layers [Fig. 7(a)] and metallic in the thick limit [Fig. 7(d)].

For the $\mathrm{LaAlO}_{3} / \mathrm{LaVO}_{3} / \mathrm{LaAlO}_{3}$ structures, there is only one polar discontinuity: the polar $\mathrm{LaAlO}_{3}$ surface. For a $\mathrm{LaVO}_{3}$ layer embedded in $\mathrm{LaAlO}_{3}$ far from any surface, there are no discontinuities to resolve, and thus no reconstructions induced. Only when the $\mathrm{LaVO}_{3}$ layer is brought in proximity to the polar $\mathrm{LaAlO}_{3}$ surface does it provide an alternative reconstruction mechanism by $\mathrm{V}$ valence changes. Therefore conductivity appears only in the thin limit, which is opposite to that for the $\mathrm{LaAlO}_{3} / \mathrm{SrTiO}_{3}$ interface.

\footnotetext{
*higuchi@hwang.k.u-tokyo.ac.jp

${ }^{1}$ M. Imada, A. Fujimori, and Y. Tokura, Rev. Mod. Phys. 70, 1039 (1998)

${ }^{2}$ A. Ohtomo and H. Y. Hwang, Nature (London) 427, 423 (2004).

${ }^{3}$ S. Okamoto and A. J. Millis, Nature (London) 428, 630 (2004).

${ }^{4}$ W.-C. Lee and A. H. MacDonald, Phys. Rev. B 74, 075106 (2006).

${ }^{5}$ G. A. Baraff, J. A. Appelbaum, and D. R. Hamann, Phys. Rev. Lett. 38, 237 (1977).

${ }^{6}$ W. A. Harrison, E. A. Kraut, J. R. Waldrop, and R. W. Grant, Phys. Rev. B 18, 4402 (1978).

${ }^{7}$ N. Nakagawa, H. Y. Hwang, and D. A. Muller, Nature Mater. 5, 204 (2006)

${ }^{8}$ M. Huijben, G. Rijnders, D. H. A. Blank, S. Bals, S. V. Aert, J. Verbeeck, G. V. Tendeloo, A. Brinkman, and H. Hilgenkamp, Nature Mater. 5, 556 (2006).

${ }^{9}$ S. Thiel, G. Hammerl, A. Schmehl, C. W. Schneider, and J. Mannhart, Science 313, 1942 (2006).
}

${ }^{10}$ A. Kalabukhov, R. Gunnarsson, J. Börjesson, E. Olsson, T. Claeson, and D. Winkler, Phys. Rev. B 75, 121404(R) (2007).

${ }^{11}$ W. Siemons, G. Koster, H. Yamamoto, W. A. Harrison, G. Lucovsky, T. H. Geballe, D. H. A. Blank, and M. R. Beasley, Phys. Rev. Lett. 98, 196802 (2007).

${ }^{12}$ D. A. Muller, N. Nakagawa, A. Ohtomo, J. L. Grazul, and H. Y. Hwang, Nature (London) 430, 657 (2004), and references therein.

${ }^{13}$ H. Y. Hwang, Science 313, 1895 (2006).

${ }^{14}$ J. Yao, P. B. Merrill, S. S. Perry, D. Marton, and J. W. Rabalais, J. Chem. Phys. 108, 1645 (1998).

${ }^{15}$ R. J. Francis, S. C. Moss, and A. J. Jacobson, Phys. Rev. B 64, 235425 (2001)

${ }^{16}$ C. H. Lanier, J. M. Rondinelli, B. Deng, R. Kilaas, K. R. Poeppelmeier, and L. D. Marks, Phys. Rev. Lett. 98, 086102 (2007).

${ }^{17}$ S. Miyasaka, T. Okuda, and Y. Tokura, Phys. Rev. Lett. 85, 5388 (2000).

${ }^{18}$ T. Ohnishi, K. Takahashi, M. Nakamura, M. Kawasaki, M. 
Yoshimoto, and H. Koinuma, Appl. Phys. Lett. 74, 2531 (1999).

${ }^{19}$ Y. Hotta, Y. Mukunoki, T. Susaki, H. Y. Hwang, L. Fitting, and D. A. Muller, Appl. Phys. Lett. 89, 031918 (2006).

${ }^{20}$ L. Fitting Kourkoutis, Y. Hotta, T. Susaki, H. Y. Hwang, and D. A. Muller, Phys. Rev. Lett. 97, 256803 (2006).

${ }^{21}$ C. Cen, S. Thiel, G. Hammerl, C. W. Schneider, K. E. Andersen, C. S. Hellberg, J. Mannhart, and J. Levy, Nature Mater. 7, 298 (2008).

${ }^{22}$ V. Vonk, M. Huijben, K. J. I. Driessen, P. Tinnemans, A. Brinkman, S. Harkema, and H. Graafsma, Phys. Rev. B 75, 235417 (2007).

${ }^{23}$ Y. Hotta, T. Susaki, and H. Y. Hwang, Phys. Rev. Lett. 99, 236805 (2007).
${ }^{24}$ M. Sayer, R. Chen, R. Fletcher, and A. Mansingh, J. Phys. C 8, 2059 (1975).

${ }^{25}$ J. B. Webb and M. Sayer, J. Phys. C 9, 4151 (1976).

${ }^{26}$ P. Dougier and P. Hagenmuller, J. Solid State Chem. 15, 158 (1975).

${ }^{27}$ H. C. Nguyen and J. B. Goodenough, Phys. Rev. B 52, 8776 (1995).

${ }^{28}$ G. Jackeli and G. Khaliullin, Phys. Rev. Lett. 101, 216804 (2008).

${ }^{29}$ Y. Hotta, H. Wadati, A. Fujimori, T. Susaki, and H. Y. Hwang, Appl. Phys. Lett. 89, 251916 (2006).

${ }^{30}$ H. Wadati et al., Phys. Rev. B 77, 045122 (2008). 